\title{
The Number of Geometric Bistellar Neighbors of a Triangulation*
}

\author{
J. A. de Loera, ${ }^{1}$ F. Santos, ${ }^{2}$ and J. Urrutia ${ }^{3}$ \\ ${ }^{1}$ The Geometry Center and School of Mathematics, University of Minnesota, \\ Minneapolis, MN 55454, USA \\ deloera@geom.umn.edu \\ ${ }^{2}$ Mathematical Institute, Oxford University, \\ 24-29 St. Giles', Oxford, Oxon OX1 3LB, England \\ ${ }^{3}$ Department of Computer Sciences, University of Ottawa, \\ Ottawa, Ontario, Canada K1N 6N5 \\ jorge@csi.UOttawa.CA
}

\begin{abstract}
The theory of secondary and fiber polytopes implies that regular (also called convex or coherent) triangulations of configurations with $n$ points in $\mathbf{R}^{d}$ have at least $n-d-1$ geometric bistellar neighbors. Here we prove that, in fact, all triangulations of $n$ points in $\mathbf{R}^{2}$ have at least $n-3$ geometric bistellar neighbors.

In a similar way, we show that for three-dimensional point configurations, in convex position and with no three points collinear, all triangulations have at least $n-4$ geometric bistellar flips. In contrast, we exhibit three-dimensional point configurations, with a single interior point, having deficiency on the number of geometric bistellar flips. A lifting technique allows us to obtain a triangulation of a simplicial convex 4-polytope with less than $n-5$ neighbors. We also construct a family of point configurations in $\mathbf{R}^{3}$ with arbitrarily large flip deficiency.
\end{abstract}

\section{Introduction}

By a triangulation of a finite point configuration $\mathcal{A}$ we mean a geometrically realized simplicial complex, all of whose vertices belong to $\mathcal{A}$ and the union of whose simplices covers the convex hull $\operatorname{conv}(\mathcal{A})$ of $\mathcal{A}$. In this note we are interested in topological

\footnotetext{
* This research was partially supported by the National Science Foundation, through a grant to the Geometry Center and an HCM postdoctoral fellowship of the European Community through a contract with the Mathematical Institute. The second authors current address is Depto. de Matemáticas, Estadistica y Computación, Universidad de Cantabria, E-39071 Santander, Spain. santos@matesco.unican.es.
} 
operations that connect two triangulations of $\mathcal{A}$. Informally, they consist of finding a subcomplex of the triangulation, which is isomorphic to part of the "lower" boundary of a simplex, and then replacing it with the "upper" boundary of the same simplex (see precise definition below). Because of the importance of a notion of adjacency of triangulations of a manifold in several different topics, the set of triangulations of a point configuration has been assigned this kind of topological operation (see, for instance, [1], [7], [11], and [13]). The topological operation we treat here arises naturally when studying the triangulations of convex polytopes within the context of the theory of secondary and fiber polytopes [2], [8], [14] and summarizes, at least partially, previous definitions.

Following the terminology of matroid theory, we call a minimal affine dependent subset of the points in $\mathcal{A}$ a circuit (see [3] or [19] for details). Every circuit $Z \subset \mathcal{A}$ admits a unique Radon partition $Z=Z_{+} \cup Z_{-}$such that the relative interiors of the convex hulls of $Z_{+}$and $Z_{-}$intersect in a point. We normally represent circuits as pairs $\left(Z_{+}, Z_{-}\right)$. If $k$ and $l$ are the cardinalities of $Z_{+}$and $Z_{-}$we say that the circuit is of type $(k, l)$.

The convex hull of a circuit $Z=\left(Z_{+}, Z_{-}\right)$can be triangulated in two ways: $k_{+}(Z)$ as the collection of $\operatorname{simplices}\left\{\operatorname{conv}(Z-\{p\}) \mid p \in Z_{+}\right\}$and $k_{-}(Z)=\{\operatorname{conv}(Z-\{p\}) \mid p \in$ $\left.Z_{-}\right\}$. Note that $k_{+}(Z)$ and $k_{-}(Z)$ resemble the bottom and top of a simplex of dimension $|Z|-1$, when viewed from antipodal points of view. We denote by $A * B$ the join of two simplicial complexes $A$ and $B$ [9].

Let $K$ be a triangulation of $\mathcal{A}$ and $Z \subset \mathcal{A}$ a circuit. We say that $K$ is supported on $Z$ if the following conditions are satisfied:

1. One of the triangulations $k_{+}(Z)$ or $k_{-}(Z)$ is a subcomplex of $K$.

2. Let $F_{1}, F_{2}, \ldots, F_{r}$ be the maximal dimensional simplices of $k_{+}(Z)$ (similarly $k_{-}(Z)$ ); then the link of $F_{i}$ in $K$ is the same simplicial complex $L$ for all $i=$ $1, \ldots, r$.

We observe that if a triangulation $K$ is supported on the circuit $Z$, then we obtain a new triangulation of $\mathcal{A}$, as follows: replace the complex $k_{+}(Z) * L$, with the complex $k_{-}(Z) * L$ (or vice versa). This operation of changing the triangulation is called a rigid bistellar flip or geometric bistellar operation. We say that the flip is of type $(k, l)$ if $Z_{+}$and $Z_{-}$have $k$ and $l$ points, respectively; equivalently, if the complex $k_{+}(Z)$ to be eliminated has $k$ maximal simplices and the complex $k_{-}(Z)$ to be introduced has $l$ maximal simplices.

Note that the usual notion of flip or diagonal exchange studied in the computational geometry literature for planar triangulations (see, for instance, [7] and [10]) is extended (corresponds to flips of type $(2,2)$ ). Points of a configuration appear or disappear in flips of types $(1, k)$ and $(k, 1)$, respectively and the most complicated flips are determined by low-dimensional affine dependences; in particular we do not assume that the points are in general position. Parts (a), (b), and (c) of Fig. 1 show the three possible types of flip in dimension 2 , which are suported in circuits of type $(2,1),(3,1)$, and $(2,2)$ respectively. Parts (d) and (e) show the two types in dimension 3 and general position, supported on circuits of type $(4,1)$ and $(3,2)$ respectively.

It remains an open question whether any two triangulations of $\mathcal{A}$ are connected by geometric bistellar flips. At present the geometric bistellar connectivity is known only in a few cases (see [11], [12], and [14]). In this context a natural question is: Can there 
(a)

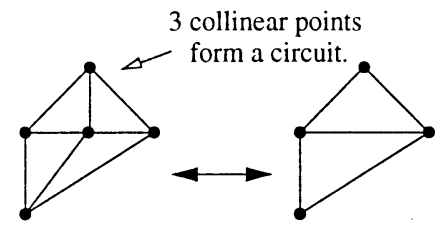

(b)

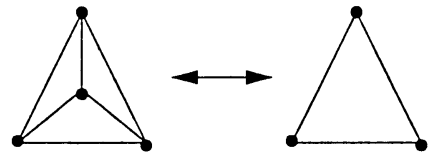

(c)

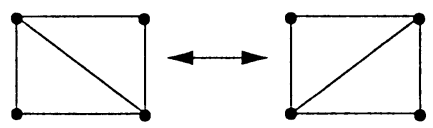

(d)

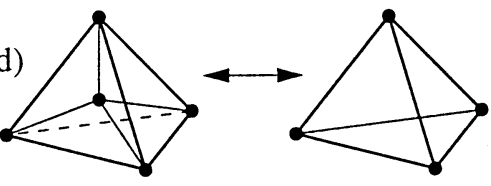

(e)

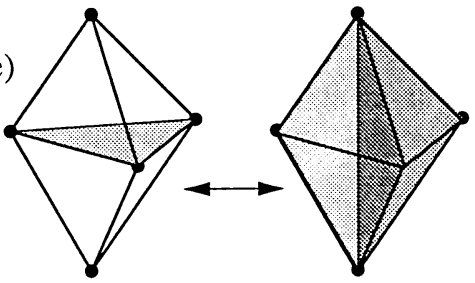

Fig. 1. Examples of geometric bistellar flips.

be a triangulation without geometric bistellar flips? Is there a triangulation with fewer geometric bistellar neighbors than the regular triangulations? We answer the second question affirmatively. At the same time, our results indicate that although it is possible that the triangulations of some three-dimensional convex polytope are disconnected, it is impossible to have isolated triangulations that contain no bistellar flips. The next theorem is a summary of our results.

\section{Theorem 1.1.}

1. Every triangulation of an $n$ point configuration in the plane has at least $n-3$ geometric bistellar flips.

2. Every triangulation of a point configuration with $n$ points in convex position, no three points collinear, has at least $n-4$ geometric bistellar flips.

3. For each value of $n$ there is a configuration in $\mathbf{R}^{3}$ with $5 n$ points and having only $3 n-2$ geometric bistellar flips.

4. There exist triangulations of simplicial 4-polytopes with $n$ vertices and fewer than $n-5$ geometric bistellar flips.

If $T$ is a triangulation of $n$ points in dimension $d$, we say that $T$ has flip deficiency if $T$ admits less than $n-d-1$ geometric bistellar flips. In Section 2 we present the proofs of items 1 and 2 of Theorem 1.1. Section 3 contains several examples which in particular prove parts 3 and 4 .

\section{Two Cases without Flip Deficiency}

The topological structure of the space of polyhedral subdivisions of an $n$ point configuration in $\mathbf{R}^{2}$ was studied by Edelman and Reiner [6]. They proved that the complex of subdivisions (ordered by refinement) has the homotopy type of a sphere of dimension 
$n-3$. Here we prove a quantitative result about the geometric bistellar neighbors, not included in that analysis.

Theorem 2.1. Every triangulation of an $n$ point configuration in the plane has at least $n-3$ geometric bistellar flips.

Proof. Let $T$ be a triangulation of an $n$ point configuration $\mathcal{A}$ in $\mathbf{R}^{2}$. If there is a flip that inserts a point $P$, then $T$ can be considered as a triangulation of $\mathcal{A} \backslash\{P\}$ and induction on $n$ shows that it has at least $n-4$ other flips. Hence we assume that the triangulation uses all the points of $\mathcal{A}$.

We say that an edge of $T$ is flippable if it is interior (not contained in the boundary of $\mathcal{A}$ ) and the two triangles incident to it form either a strictly convex quadrilateral or a quadrilateral with two consecutive edges whose union is a straight line segment contained in the boundary of the convex hull of $\mathcal{A}$. In the first case there is a flip of type $(2,2)$ which removes the flippable edge and inserts the other diagonal of the quadrilateral, and in the second case there is a flip of type $(2,1)$ which removes the interior edge and joins the two consecutive collinear edges into one (corresponding to the upper half of Fig. 1(a)).

Let $e_{b}$ be the number of boundary edges (note that $e_{b}$ also equals the number of boundary points of $\mathcal{A}$ ). Denote by $e_{i}$ the number of interior edges and by $f$ the number of triangles. Euler's formula for the disk gives $n-e_{i}-e_{b}+f=1$ and a counting argument shows that $3 f=2 e_{i}+e_{b}$. With these two equalities we obtain

$$
e_{i}=3 n-3-2 e_{b} \text {. }
$$

For an interior nonflippable edge $a$, the union of the two triangles sharing $a$ is a quadrangle with a concave or flat vertex which we call the vertex associated to $a$. If a vertex $p$ is associated to four interior edges, then the four edges form two pairs of collinear edges with $p$ as a common end and there are two flips of type $(2,1)$ which make $p$ disappear. If $p$ is associated to three interior edges, then the star of $p$ looks like either part (a) or (b) of Fig. 1, and there is one flip (of type $(2,1)$ or $(3,1)$ ) which makes the point $p$ disappear.

Hence, the number of interior nonflippable edges is no greater than twice the number of interior points plus the number of flips which make a point disappear. In other words, the total number $e_{i}$ of interior edges is no greater than the total number of flips plus twice the number $n-e_{b}$ of interior points. Thus the number of flips is at least $e_{i}-2\left(n-e_{b}\right)=n-3$, as desired.

Now we consider the case of three-dimensional point configurations. Let $T$ be a triangulation of a point configuration $\mathcal{A}$ in $\mathbf{R}^{3}$ in convex position. By this we mean that all the points of $\mathcal{A}$ are in the boundary of $\operatorname{conv}(\mathcal{A})$. We further assume that no three points are collinear and that $T$ uses all the points of $\mathcal{A}$. In this case the possible flips that may appear are of types $(3,2),(2,3),(2,2)$, and $(3,1)$, but the latter appears with the restriction that all four points lie in the same facet of the boundary of $\operatorname{conv}(\mathcal{A})$; otherwise the interior point of the circuit is in the interior of $\operatorname{conv}(\mathcal{A})$, contradicting the convex position assumption.

Consider the union of two tetrahedra $A:=[p, q, r, s]$ and $B:=[q, r, s, t]$. We say that the edge $[q, r]$ of the common triangle $[q, r, s]$ is convex, flat, or concave for the 
pair $(A, B)$, or for the triangle $[q, r, s]$, if the dihedral angle of $A \cup B$ at $[q, r]$ is less than, equal to, or greater than $180^{\circ}$, respectively.

The proof that $T$ has at least $n-4$ flips follows the same lines as the proof of the two-dimensional case, but more cases have to be considered. We divide the flips of $T$ into triangle-flips and edge-flips according to the following:

(a) A flip is called a triangle-flip if there is only one interior triangle $c$ of $T$ that disappears as a result of the flip. We say that the triangle is flippable. This comprises the following two cases:

- A $(2,3)$ flip. The two tetrahedra incident to $c$ form a convex bipyramid. The flip removes $c$ and inserts the axis of the bipyramid, as well as three triangles incident to the axis.

- A $(2,2)$ flip with the circuit in a facet of $\operatorname{conv}(\mathcal{A})$. The bipyramid in question has only one nonconvex edge that is flat and lies in the boundary of $\operatorname{conv}(\mathcal{A})$. The flip removes $c$ and the flat edge and inserts the other diagonal of the quadrilateral formed in the facet, as well as a triangle incident to it.

(b) A flip is called an edge-flip if it is not a triangle-flip and there is only one interior edge $e$ of $T$ that disappears as a result of the flip. We say that $e$ is a flippable edge. This comprises the following cases:

- A $(3,2)$ flip: the star of $e$ is a convex triangular bipyramid with $e$ as axis, triangulated with the three tetrahedra that have $e$ as an edge. The flip removes $e$ and the three triangles incident to it and inserts a triangle which intersects $e$.

- A $(3,1)$ flip with the circuit in a facet of $\operatorname{conv}(\mathcal{A})$. The flip removes the edge $e$ as well as the three tetrahedra incident to it, that now form a triangular pyramid with one of the extremes of $e$ as apex and the other in the interior of the base. The flip removes $e$ as well as the three tetrahedra incident to it and puts the pyramid instead.

- A $(2,2)$ flip with the circuit not in a facet of $\operatorname{conv}(\mathcal{A})$. The star of $e$ has four tetrahedra and at least two of the four triangles incident to $e$ form a planar convex quadrilateral. The flip removes $e$ and inserts the other diagonal of the quadrilateral. It is like two triangle-flips of type $(2,2)$ performed at the same time.

Theorem 2.2. Every triangulation of an $n$ point configuration $\mathcal{A} \subset \mathbf{R}^{3}$ in convex position, without collinearities, has at least $n-4$ geometric bistellar flips.

Proof. Let $T$ be a triangulation of an $n$ point configuration $\mathcal{A}$ in $\mathbf{R}^{3}$, in convex position. Again, if $T$ does not use a certain point $a \in \mathcal{A}$, then it has at least $n-5$ flips as a triangulation of $\mathcal{A} \backslash\{a\}$ plus the flip which inserts the point $a$. Thus, we assume that $T$ uses all the vertices.

Let $t$ denote the number of tetrahedra and let $f_{i}$ and $f_{b}$ (resp. $e_{i}$ and $e_{b}$ ) be the numbers of interior and boundary triangles (resp. edges).

Euler's formulas for the 3-ball and the 2-sphere imply

$$
f_{i}+f_{b}+n=t+e_{i}+e_{b}+1, \quad f_{b}+n=e_{b}+2 .
$$

We also have

$$
2 f_{i}+f_{b}=4 t, \quad 2 e_{b}=3 f_{b} .
$$


Putting these equations together we obtain

$$
e_{i}=t-n+3, \quad f_{i}=2 t-n+2 \text {. }
$$

As a consequence we have the following important relation:

$$
f_{i}-2 e_{i}=n-4 \text {. }
$$

In an analogous way to the proof of Theorem 2.1, we associate the interior nonflippable triangles of $T$ to certain interior edges of $T$ and then use a counting argument to conclude that the number of flips is at least $n-4$. To formalize this, we consider each nonflippable interior triangle as having a "unit of charge," which we transfer to one of its three edges. Observe that if $c$ is a nonflippable interior triangle and $A$ and $B$ are the tetrahedra sharing it, then at least one of the edges of $c$ is flat or concave in $A \cup B$. There are two cases:

1. If one of the edges of $c$ is concave in $A \cup B$, then it is interior and is the only concave edge of the pair $(A, B)$, because if there are two concave edges their common endpoint cannot be in the boundary of $\operatorname{conv}(\mathcal{A})$. We give the charge of $c$ to this concave edge.

2. Otherwise there is a unique nonconvex edge of $c$ which is flat for the pair $(A, B)$, because two flat edges would imply the existence of three collinear points. Moreover, the flat edge is interior because otherwise $c$ is flippable according to the discussion above. We give the charge of $c$ to the flat edge.

We claim that the total charge of the interior edges is at most twice the number of interior edges plus the number of edge-flips. From the claim it follows that the total number of interior triangles $f_{i}$ is at most the total number of flips plus twice the number of interior edges $e_{i}$. Thus, the number of flips is at least $f_{i}-2 e_{i}=n-4$, as desired.

To prove our claim, we only need to analyze interior edges with charge greater than two:

(A) If an interior edge $[p, q]$ is a nonconvex edge for at least four pairs of consecutive tetrahedra in its star, then the star has precisely four tetrahedra and the sum of any two consecutive dihedral angles is 180 . Let $a, b, c$, and $d$ be the four points in the link of $[p, q]$, in circular order. The planar quadrilaterals $[a, p, c, q]$ and $[b, p, d, q]$ are strictly convex; otherwise one of the points $p$ or $q$ would either be collinear with two of $a, b, c$, and $d$ or either $p$ or $q$ would be contained in the convex hull of the other five points (violating the convexity assumption). Thus, the star of $[p, q]$ forms a convex octahedron. The charge associated to $[p, q]$ is four (the four triangles containing $[p, q]$ are as in part (2) above) and there are two edge-flips of type $(2,2)$ which make the edge $[p, q]$ disappear, supported in the circuits $(\{p, q\},\{a, c\})$ and $(\{p, q\},\{b, d\})$.

(B) If an interior edge $[p, q]$ is nonconvex for exactly three pairs of consecutive tetrahedra in its star, then there can be three or four tetrahedra in the star of $[p, q]$.

If there are three, then $[p, q]$ is a concave edge associated to the three interior triangles incident to it. Let $a, b$, and $c$ be the three vertices in the link of $[p, q]$. Then either $(\{a, b, c\},\{p, q\})$ is a $(3,2)$ circuit or one of $(\{a, b, c\},\{p\})$ and 
( $\{a, b, c\},\{q\})$ is a $(3,1)$ circuit with $a, b$, and $c$ lying in a facet of $\mathcal{A}$. In either case, there is an edge-flip supported in the circuit which makes $[p, q]$ disappear.

If there are four, then let $a, b, c$, and $d$ be the four points in the link of $[p, q]$ in circular order. Two of them (say $a$ and $c$ ) are coplanar with $[p, q] .[p, q]$ is a concave edge associated with one of the triangles $[p, q, a]$ or $[p, q, c]$ and a planar edge for the triangles $[p, q, b]$ and $[p, q, d]$. Note that the triangles $[p, q, b]$ and $[p, q, d]$ are in the situation of (2) above, with their charges associated to $[p, q]$. $[p, q]$ has a charge of three, but $(\{a, c\},\{p, q\})$ is a $(2,2)$ circuit and there is an edge-flip supported on it which makes $[p, q]$ disappear.

(C) If an interior edge $[p, q]$ is nonconvex for at most two pairs of consecutive tetrahedra in its star, then the charge of $[p, q]$ is at most two.

\section{Remark 2.3.}

- A more elaborate argument makes it possible to eliminate the hypothesis that no three points are collinear. The idea is again to distribute the charges of nonflippable interior triangles to the edges of the triangulation, but with flips of type $(2,1)$ present we have to distribute the charges to boundary edges as well.

- Contained in the above theorem is the case when $\mathcal{A}$ is the set of vertices of a convex 3 -polytope. Thus, for example, we can guarantee for the Platonic solids that every triangulation of the icosahedron has at least eight flips, every triangulation of the dodecahedron has at least sixteen flips and every triangulation of the cube has at least four flips.

\section{Constructions with Flip Deficiency}

Let $P_{1}$ and $P_{2}$ be two triangular prisms with a square face of the same size. We glue them together over the square face, making sure to rotate $P_{2} 90^{\circ}$, so that triangular faces of $P_{1}$ are adjacent to square faces of $P_{2}$ and vice versa (see Fig. 2).

We call this polytope a biprism. The configuration $\mathcal{A}$ of our interest is given by the eight vertices of the biprism together with the center of their common square facet. Possible coordinates for the nine points are given as columns of the following matrix:

$$
\mathcal{A}=\left[\begin{array}{ccccccccc}
0 & 0 & 0 & 1 & -1 & 1 & 1 & -1 & -1 \\
0 & -1 & -1 & 1 & 1 & 0 & 0 & 0 & 0 \\
0 & 1 & -1 & 0 & 0 & 1 & -1 & 1 & -1
\end{array}\right]
$$

We refer to the points by their indices. In this way the facets of the biprism are $[5,8,9]$, $[4,6,7],[3,7,9],[2,6,8],[4,5,6,8],[2,3,6,7],[2,3,8,9]$, and $[4,5,7,9]$.

Triangulate the boundary of the biprism by inserting one of the two diagonals of each square facet in such a way that all the vertices belong to a diagonal. There are two ways of doing this, equivalent by a symmetry of the biprism. Figure 2 shows one of them. Consider the triangulation of $\mathcal{A}$ consisting of the cones over the triangles of the boundary with apex at the central point. More precisely, let the maximal simplices of $T$ be $\{[1,4,7,9],[1,5,6,8],[1,5,8,9],[1,3,7,9],[1,3,8,9],[1,4,5,6],[1,4,5,9]$, $[1,4,6,7],[1,2,3,7],[1,2,3,8],[1,2,6,7],[1,2,6,8]\}$. 


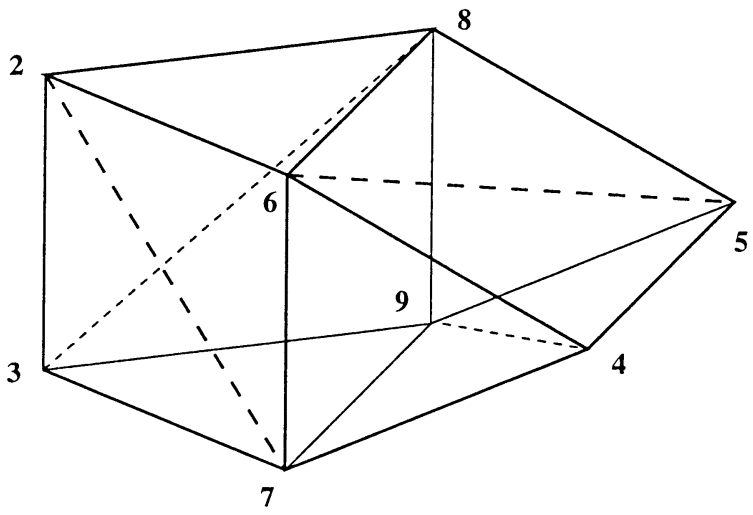

Fig. 2. A triangulated biprism.

It is clear that this triangulation has at least four flips supported on the four quadrilateral facets. In fact, these are the only flips, but we prove this only after a perturbation.

Theorem 3.1. There is a triangulation of a generic three-dimensional configuration with nine points, only one of them interior, with only four geometric bistellar flips.

Proof. We perturb the above example, whose points are not in general position. All coplanar quadruples involve two of the points $6,7,8,9$. We slightly modify their $y$ coordinates, by a sufficiently small value $\varepsilon$, in order to have the points in general position:

$$
\left[\begin{array}{ccccccccc}
0 & 0 & 0 & 1 & -1 & 1 & 1 & -1 & -1 \\
0 & -1 & -1 & 1 & 1 & -\varepsilon & \varepsilon & \varepsilon & -\varepsilon \\
0 & 1 & -1 & 0 & 0 & 1 & -1 & 1 & -1
\end{array}\right]
$$

Take for instance $\varepsilon=\frac{1}{10}$. The perturbation makes the simplicial complex $T$ nonconvex. We complete it to a convex simplicial complex $T^{\prime}$ with the four new simplices $[2,3,8,9],[4,5,6,8],[2,3,6,7]$, and [4, 5, 7, 9], which replace the former square facets of the biprism. We claim that there are only four flips. A priori there are three possible types of flips: $(4,1)$ flips removing the interior point, $(2,3)$ flips, and $(3,2)$ flips. Removing the interior point by a flip is impossible because it is a vertex of more than four tetrahedra. Flips of type $(3,2)$ are in bijection with interior edges surrounded by exactly three tetrahedra. There are four such edges corresponding to the former diagonals of the squares of the biprism. Any other interior edge contains the origin and is surrounded by four or five tetrahedra. Flips of type $(2,3)$ are in bijection with interior triangles incident to two tetrahedra, which form a convex bipyramid. Up to symmetry there are only seven types of interior triangles, with representatives [2, 3, 7], [2, 6, 7], $[1,2,7],[1,2,3],[1,2,6],[1,2,8]$, and $[1,6,7]$. None of them gives rise to a convex bipyramid. 


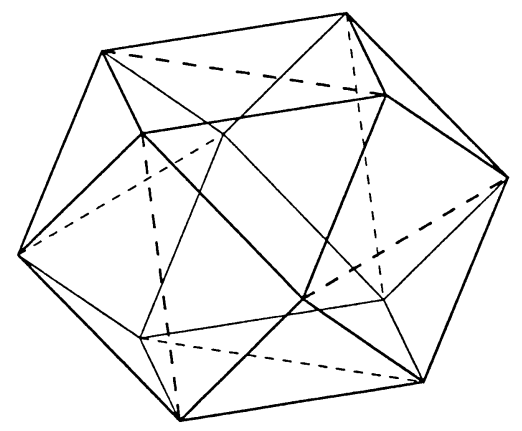

Fig. 3. A triangulated cuboctahedron with an interior point.

Remark 3.2. Another example, with larger flip deficiency, can be constructed from the regular cuboctahedron with equal length edges; i.e., the convex hull of the midpoints of the edges of a regular 3-cube. Consider the thirteen point configuration consisting of the twelve vertices of the cuboctahedron and the center point. Triangulate the boundary of the cuboctahedron by inserting a diagonal in each of the squares in a way that every vertex belongs to precisely one diagonal (as shown in Fig. 3). Cone the triangulation of the boundary with the center point, as we did with the biprism. The triangulation obtained has only six flips, supported on the square facets of the cuboctahedron. This gives a flip deficiency of three. Moreover, the points can be perturbed into general position without increasing the number of flips of the triangulation (for this, "enlarge" each of the six diagonals a little bit and then perturb in an arbitrary way).

We have detailed the biprism example because, to our knowledge, it is the smallest point configuration with fewer geometric flips than the dimension of the secondary polytope. This is interesting, since even more complex nonregular triangulations, such as Rudin's nonshellable triangulation [15], do not have this property [18]. This can also be verified using the program PUNTOS for the study of secondary polytopes and triangulations [4].

The Lawrence construction (see p. 386 of [3] or p. 180 of [19]) is a typical tool for translating results of point configurations to convex polytopes, but it does not preserve triangulations of a configuration to its lifting. In the following proposition we show a general lifting construction, somewhat similar to the Lawrence construction, which allows us to do this. See [16] for a further exploration of the lifting procedures and triangulations.

Proposition 3.3. Let $\mathcal{A}$ be a configuration with $n$ points, $k$ of them interior, in dimension $d$. There exists a point configuration $\mathcal{A}^{\prime}$ with $n+1$ points, $k-1$ of them interior, in dimension $d+1$ with the following property: there is a bijection between triangulations of $\mathcal{A}$ and triangulations of $\mathcal{A}^{\prime}$ which preserves geometric bistellar adjacency. Moreover, if the convex hull of $\mathcal{A}$ is simplicial, then the convex hull of $\mathcal{A}^{\prime}$ is also simplicial. 
Proof. Let $P$ be an interior point of $\mathcal{A} \subset \mathbf{R}^{d}$. Consider the new configuration

$$
\mathcal{A}^{\prime}=\{(Q, 0), \text { for } Q \in \mathcal{A}, Q \neq P\} \cup\{(P, 1),(P,-1)\} \subset \mathbf{R}^{d+1} .
$$

The configuration $\mathcal{A}^{\prime}$ has $n+1$ points, dimension $d+1$, and the points $(P, 1),(P,-1)$ are on the boundary of $\operatorname{conv}\left(\mathcal{A}^{\prime}\right)$. The boundary of $\operatorname{conv}\left(\mathcal{A}^{\prime}\right)$ equals the suspension over the boundary of $\operatorname{conv}(\mathcal{A})$; i.e., $\operatorname{conv}\left(\mathcal{A}^{\prime}\right)$ is a bipyramid over $\operatorname{conv}(\mathcal{A})$. Moreover, if $\operatorname{conv}(\mathcal{A})$ is simplicial, so is $\operatorname{conv}\left(\mathcal{A}^{\prime}\right)$.

For every triangulation $T$ of $\mathcal{A}$ consider the following collection $T^{\prime}$ of simplices of $\mathcal{A}^{\prime}$ : for a simplex $\sigma \in T$ which does not contain $P$ as a vertex, $T^{\prime}$ contains the simplices $\sigma *(P, 1)$ and $\sigma *(P,-1)$. For a simplex $\tau * P \in T$ which contains $P$ as a vertex, $T^{\prime}$ contains the simplex $\tau *[(P, 1),(P,-1)]$. Then $T^{\prime}$ is a triangulation of $\mathcal{A}^{\prime}$. We claim that all the triangulations of $\mathcal{A}^{\prime}$ arise in this way. In fact, it follows from Section 5 in [5] (in particular Theorem 5.4) that the triangulations of a point configuration can be characterized by the real or virtual chambers of its Gale transform (for a definition of Gale transform, see also [17] or p. 168 of [19]). On the other hand, the Gale transform of $\mathcal{A}^{\prime}$ is the same as that of $\mathcal{A}$, but with the vector corresponding to $P$ repeated twice. Thus, the two Gale transforms have the same chambers.

Also, all the circuits of $\mathcal{A}^{\prime}$ arise in the following way: if $Z=Z_{+} \cup Z_{-}$is a circuit of $\mathcal{A}$ not containing $P$, then $Z$ is also a circuit of $\mathcal{A}^{\prime}$. If $Z=Z_{+} \cup Z_{-}$is a circuit of $\mathcal{A}$ with $P \in Z_{+}$, then $Z^{\prime}:=(Z \backslash P) \cup\{(P, 1),(P,-1)\}$ is a circuit of $\mathcal{A}^{\prime}$ with positive part $\left(Z_{+} \backslash P\right) \cup\{(P, 1),(P,-1)\}$ ) and negative part $Z_{-}$(with the obvious analogue if $P \in Z_{-}$). From here it follows that two triangulations of $\mathcal{A}^{\prime}$ are geometric bistellar neighbors if and only if the corresponding triangulations of $\mathcal{A}$ are geometric bistellar neighbors.

Corollary 3.4. There are simplicial 4-polytopes with ten and fourteen points, respectively, and triangulations of them having four and six geometric bistellar flips, respectively.

Proof. Apply Proposition 3.3 to the point configuration exhibited in Theorem 3.1 and (a perturbed version of) the one in Remark 3.2.

Another question, particularly natural due to the open problem of connectivity by geometric bistellar flips, is whether the ratio of the number of bistellar neighbors divided by the number of vertices can be made arbitrarily small as the number of vertices of a polytope grow. Although we do not know of a construction that could yield this, we present the following theorem:

Theorem 3.5. For each value of $n$ there is a configuration with $5 n$ points in $\mathbf{R}^{3}$ having only $3 n-2$ geometric bistellar flips.

Proof. We provide an inductive construction which relies on stacking successive layers of points. For $n=1$ let $\mathcal{A}_{1}$ be the vertices of the bipyramid over a triangle: for example, $a_{1}:=(1,0,0), b_{1}:=(0,1,0), c_{1}:=(-1,-1,0), d_{1}:=(0,0,1), e_{1}:=(0,0,-1)$. We triangulate $\mathcal{A}_{1}$ by two tetrahedra $\left[a_{1}, b_{1}, c_{1}, d_{1}\right]$ and $\left[a_{1}, b_{1}, c_{1}, e_{1}\right]$. 


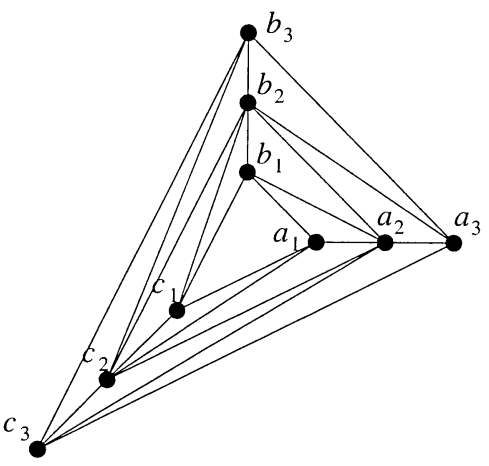

(a)

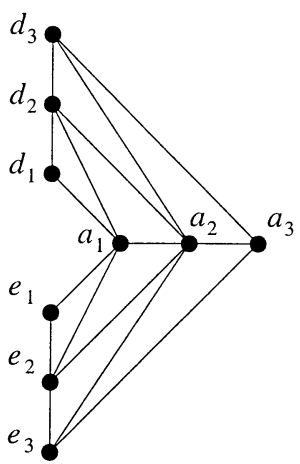

(b)

Fig. 4. The triangulation of $\mathcal{A}_{3}$.

The vertices of the $n$th step of the construction are defined as follows, where $m \mathcal{A}_{1}$ denotes the dilation of $\mathcal{A}_{1}$ by a scalar factor $m$ :

$$
\mathcal{A}_{n}=\mathcal{A}_{1} \cup 2 \mathcal{A}_{1} \cup \cdots \cup n \mathcal{A}_{1} .
$$

We use the notation $a_{i}, b_{i}, c_{i}, d_{i}, e_{i}$ for $i a_{1}, i b_{1}, i c_{1}, i d_{1}, i e_{1}$. Notice that the horizontal plane contains a nest of $n$ concentric triangles $T_{1}, \ldots, T_{n}$, where $T_{i}=\left[a_{i}, b_{i}, c_{i}\right]$. The intermediate zone in between two of these triangles will be triangulated by the well-known symmetric, whirlpool-like nonregular triangulation (which appeared, for example, on p. 219 of [8] and in [2]). Part (a) of Fig. 4 shows the resulting triangulation of the plane $z=0$ for $n=3$.

Join the triangles in between $T_{i}$ and $T_{i+1}$ to the vertices $d_{i}$ and $e_{i}$ of the $i$ th bipyramid. Finally join the edges of the triangle $T_{i}$, for $i \geq 2$, to the edges $\left[d_{i-1}, d_{i}\right]$ and $\left[e_{i-1}, e_{i}\right]$. Part (b) of Fig. 4 shows the triangles that lie in the plane $y=0$, for $n=3$. Each of these triangles will be joined to one of the $b_{i}$ and one of the $c_{i}$, in a way which is consistent with part (a).

We study the possible flips of the triangulation. There is a unique circuit contained in every five points if four of them are affinely independent.

Consider first the case of a tetrahedron having an edge in the axis. Without loss of generality we assume it to be $\sigma=\left[a_{i}, b_{i}, d_{i}, d_{i-1}\right]$, for $i \in\{2, \ldots, n\}$. Its adjacent tetrahedra are $\left[a_{i}, c_{i}, d_{i}, d_{i-1}\right],\left[b_{i}, c_{i}, d_{i}, d_{i-1}\right],\left[a_{i}, b_{i}, b_{i-1}, d_{i-1}\right]$, and $\left[a_{i}, b_{i}, a_{i+1}, d_{i}\right]$. The only circuit contained in the vertices of $\sigma$ and either of the first two tetrahedra is $\left(\left\{a_{i}, b_{i}, c_{i}, d_{i}\right\},\left\{d_{i-1}\right\}\right)$, and there is no flip supported on it because the tetrahedron $\left[d_{i-1}, a_{i}, b_{i}, c_{i}\right]$ is missing. The only circuit contained in the vertices of $\sigma$ and the third tetrahedron (resp. the fourth) is $\left(\left\{d_{i-1}, b_{i}\right\},\left\{b_{i-1}, d_{i}\right\}\right)$ (resp. $\left.\left(\left\{d_{i-1}, a_{i+1}\right\},\left\{a_{i}, d_{i}\right\}\right)\right)$ and there is no flip supported on it because the triangles $\left[b_{i}, d_{i}, d_{i-1}\right]$ and $\left[b_{i}, b_{i-1}, d_{i-1}\right]$ (resp. $\left[a_{i}, d_{i}, d_{i-1}\right]$ and $\left[a_{i}, a_{i+1}, d_{i}\right]$ ) have different links.

We now consider the case of two adjacent tetrahedra none of which has an edge on the axis. This implies that each of them has one vertex on the axis and gives the following possibilities: two tetrahedra sharing one vertex in the axis and with the other vertices (four in total) in $z=0$, or two tetrahedra sharing a triangle in $z=0$. In the first case the four points in $z=0$ are a circuit and for it to be flippable it is necessary (and 
in our construction sufficient) for the circuit to be flippable in the planar configuration of part (a) of Fig. 4. This planar configuration has $3 n-3$ flips, three of them in the layer between two concentric triangles. In the second possibility, either the triangle in $z=0$ is $\left[a_{1}, b_{1}, c_{1}\right]$, which gives rise to the flip of the bipyramid $\mathcal{A}_{1}$, or we can assume without loss of generality that the triangle contains an edge $\left[a_{i}, a_{i+1}\right]$. In this case the two points in the axis will be $d_{i}$ and $e_{i}$ (as follows from part (b) of the figure) and the circuit contained in the vertices of the two tetrahedra will be $\left(\left\{a_{i}\right\},\left\{a_{i+1}, d_{i}, e_{i}\right\}\right)$. This circuit cannot be flippable in the triangulation since the edge $\left[d_{i}, e_{i}\right]$ does not appear. Thus, the total number of flips is $3 n-2$, as desired.

\section{References}

1. J. W. Alexander, The combinatorial theory of complexes, Ann. of Math. 31 (1930), 292-320.

2. L. Billera, P. Filliman, and B. Sturmfels, Constructions and complexity of secondary polytopes, $A d v$. in Math. 83 (1990), 155-179.

3. A. Björner, M. Las Vergnas, B. Sturmfels, N. White, and G. Ziegler, Oriented Matroids, Cambridge University Press, Cambridge, 1992.

4. J. A. de Loera, Triangulations of Polytopes and Computational Algebra, Ph.D. dissertation, Cornell University, 1995.

5. J. A. de Loera, S. Hoşten, F. Santos, and B. Sturmfels, The polytope of all triangulations of a point configuration, Doc. Math. J. DMV 1 (1996), 103-119.

6. P. H. Edelman and V. Reiner, Visibility complexes and the Baues problem for triangulations in the plane, to appear in Discrete Comput. Geom.

7. H. Edelsbrunner and N. R. Shah, Incremental topological flipping works for regular triangulations, Proc. 8th Annual ACM Symp. on Computational Geometry, ACM Press, New York, 1992, pp. 43-52.

8. I. M. Gel'fand, M. M. Kapranov, and A. V. Zelevinsky, Discriminants, Resultants and Multidimensional Determinants, Birkhäuser, Boston, 1994.

9. L. Glaser, Geometrical Combinatorial Topology, Van Nostrand Reinhold, New York, 1970.

10. F. Hurtado, M. Noy, and J. Urrutia, Flipping edges on triangulations. Proc. Twelfth ACM Annual Symp. on Computational Geometry, 1996, pp. 214-223.

11. C. L. Lawson, Software for $C^{1}$-interpolation, in Mathematical Software III (John Rice, ed.), Academic Press, New York, 1977, pp. 161-194.

12. C. W. Lee, Regular triangulations of convex polytopes, in Applied Geometry and Discrete MathematicsThe Victor Klee Festschrift (P. Gritzmann and B. Sturmfels, eds.), DIMACS Series in Discrete Mathematics and Theoretical Computer Science, Vol. 4, AMS-ACM, Providence, RI, 1991, pp. 443-456.

13. U. Pachner, P. L. homeomorphic manifolds are equivalent by elementary shellings, European J. Combin. 12 (1991), 129-145.

14. J. Rambau, Triangulations of cyclic polytopes and higher Bruhat orders, Mathematika 44 (1997), 162-175.

15. M. E. Rudin, An unshellable triangulation of a tetrahedron, Bull. Amer. Math. Soc. 64 (1958), 90-91.

16. F. Santos, Triangulations of oriented matroids, manuscript, 1996.

17. B. Sturmfels, Some applications of affine Gale diagrams to polytopes with few vertices, SIAM J. Discrete Math. 1 (1988), 121-133.

18. B. Sturmfels, Private communication, 1990.

19. G. Ziegler, Lectures on Polytopes, Springer-Verlag, New York, 1994.

Received November 25, 1996, and in revised form March 10, 1997.

Note added in proof. Recently, F. Santos constructed triangulations in $R^{3}$ and $R^{4}$ with $O(\sqrt{n})$ and $O(1)$ flips, for arbitrarily large numbers $n$ of vertices. 